\title{
Effects of temporal trial-by-trial cuing on early and late stages of auditory processing: Evidence from event-related potentials
}

\author{
Alexa Lampar • Kathrin Lange
}

Published online: 18 May 2011

(C) Psychonomic Society, Inc. 2011

\begin{abstract}
Temporal-cuing studies show faster responding to stimuli at an attended versus unattended time point. Whether the mechanisms involved in this temporal orienting of attention are located early or late in the processing stream has not been answered unequivocally. To address this question, we measured event-related potentials in two versions of an auditory temporal cuing task: Stimuli at the uncued time point either required a response (Experiment 1) or did not (Experiment 2). In both tasks, attention was oriented to the cued time point, but attention could be selectively focused on the cued time point only in Experiment 2. In both experiments, temporal orienting was associated with a late positivity in the timerange of the P3. An early enhancement in the timerange of the auditory N1 was observed only in Experiment 2. Thus, temporal attention improves auditory processing at early sensory levels only when it can be focused selectively.
\end{abstract}

Keywords Attention · Evoked potentials · Temporal processing

In most everyday situations, we experience stimulation from different sensory modalities that arises from various spatial locations and changes dynamically over time. Typically, only a small subset of the information that reaches our sensory systems is relevant for our current task. We therefore choose certain stimuli for prioritized processing. Which stimuli we choose is influenced by our current needs and expectations, but we may also freely decide to

\section{A. Lampar $\cdot \mathrm{K}$. Lange $(\bowtie)$}

Department of Experimental Psychology,

Heinrich-Heine University,

40225 Düsseldorf, Germany

e-mail: kathrin.lange@uni-duesseldorf.de attend to certain stimuli. The underlying processes are typically referred to as attentional orienting. In other words,"attentional orienting can be defined as the set of processes by which neural resources are deployed selectively toward specific attributes of events on the basis of changing motivation, expectation, or volition in order to optimize perception and action" (Nobre, 2004, p. 157). Whereas the majority of attention research has focused on the spatial orienting of attention, evidence has accumulated over the last decade that perception and action can also be improved by orienting attention to specific moments in time (Nobre, Correa, \& Coull 2007; Nobre \& Coull, 2010; Nobre \& O'Reilly, 2004). Behavioral evidence (i.e., faster and more accurate responses) for temporal orienting has been provided by visual studies using a temporal-cuing paradigm, similar to the symbolic spatial-cuing task developed by Posner and colleagues (Posner, Snyder, \& Davidson, 1980). In symbolic temporal cuing, the cue indicates when (instead of where) the target will most likely appear (e.g., Correa, Lupiáñez, Milliken, \& Tudela, 2004; Correa, Lupiáñez, \& Tudela 2005; Coull \& Nobre, 1998; Griffin, Miniussi, \& Nobre, 2001, 2002; Miniussi, Wilding, Coull, \& Nobre, 1999). As in spatial cuing, it is assumed that the increased probability of targets at one time point gives rise to an attention shift to this time point. Different responding to cued and uncued stimuli is regarded as a sign of attentional orienting. Since, however, the uncued stimuli are relevant for response-selection, too, they may not be completely ignored. Hence, the attentional difference between cued and uncued conditions is smaller than in a selective attention paradigm (see below).

A central issue in attention research is whether improved responding is due to processes located at early sensory or at later stages of stimulus processing. Event-related potential (ERP) data obtained with the temporal-cuing paradigm are unequivocal with respect to this question. Some studies 
have reported effects of temporal orienting in the N2 and P3, which are associated with decision- or motor-related processing stages, but not in the sensory-evoked $\mathrm{P} 1$ and $\mathrm{N} 1$ of the visually evoked potential (Griffin et al., 2002, Experiment 2; Miniussi et al., 1999). On the other hand, there is evidence for early sensory effects. Griffin et al. (2002, Experiment 1) found the visually evoked N1 to be enhanced by temporal orienting, and Correa and colleagues reported a modulation of the visual P1 (Correa, Lupiáñez, Madrid, \& Tudela, 2006).

Additional evidence that temporal orienting can improve early sensory-processing stages is provided by auditory temporal-orienting studies. These consistently observed a modulation of early sensory processing (an enhanced N1) and of later decision- or response-related processing stages (a larger positivity in the time range of the P3; Lange, Krämer, \& Röder, 2006; Lange \& Röder, 2006; Lange, Rösler, \& Röder, 2003; Röder, Krämer, \& Lange, 2007; Sanders \& Astheimer, 2008; for a review, see Lange \& Röder, 2010). Larger negativities in the time range of the auditory N1 have also been reported by spatial attention studies (e.g., Giard, Perrin, Pernier \& Peronnet, 1988; Hillyard, Hink, Schwent, \& Picton, 1973; Näätänen, Gaillard, \& Mäntysalo, 1978; Woldorff \& Hillyard, 1991; see also Näätänen \& Picton, 1987) and have been interpreted in favor of a selection at early sensory-processing stages.

In the auditory temporal-orienting studies (Lange et al., 2006; Lange \& Röder, 2006; Lange et al., 2003; Röder et al., 2007; Sanders \& Astheimer, 2008), a paradigm different from those in the cuing studies was used, where stimuli at an unattended time point never required a response. In the temporal version of the attention paradigm introduced by Hillyard and colleagues (Hillyard et al., 1973), stimulus pairs were presented to the participants (Lange et al., 2003). The participants were instructed to attend to a particular time point after the first stimulus (600 or 1,200 ms after the first stimulus). The attended interval alternated between blocks. A response was required only if an infrequent (deviant) stimulus was presented at the attended time point. Stimuli at the unattended time were never relevant for response-selection and could be completely ignored. Attention effects were assessed by comparing the ERPs with physically identical frequent (standard) stimuli that did not require a response as a function of attention. For example, the processing of a stimulus appearing $600 \mathrm{~ms}$ after the first stimulus when this time point was attended was compared with the processing of the very same stimulus $(600 \mathrm{~ms}$ after the first stimulus) when the other time point was attended. Because, in this task, unattended stimuli never require a response, the participants may orient their attention selectively to one time point. In a cuing task, by contrast, participants have to divide their attention between cued and uncued time points, because both require a response (see also Eimer, 1994, 1996). Notably, spatial-orienting research suggests that attention effects on early sensory processes are found only if attention can be strongly focused on one location (i.e., in selective attention), but not when attention is divided between cued and uncued locations (Eimer, 1994, 1996; Schröger \& Eimer, 1997; but see Mangun \& Hillyard, 1991; Talsma, Mulckhuyse, Slagter, \& Theeuwes, 2007; Talsma, Slagter, Nieuwenhuis, Hage, \& Kok, 2005).

When unattended stimuli are not response relevant, effects of temporal orienting cannot be assessed at a behavioral level. Hence, the functional significance of the early auditory effects of temporal orienting has not been evaluated so far. The goal of the present study was thus twofold. First, we wanted to provide evidence for the functional validity of auditory temporal orienting - that is, demonstrate improved stimulus processing on a behavioral level. Second, we investigated whether modulations at sensory-processing levels are observed when attention is not selectively oriented to a time point but, rather, divided between a cued and an uncued moment.

We developed an auditory temporal-cuing paradigm similar to that used in earlier visual studies (e.g., Miniussi et al., 1999). In our study, a high or a low tone predicted the onset of an auditory target (white noise burst) after one of two intervals (stimulus onset asynchronies [SOAs] of 600 and 1,200 ms; see Fig. 1). The target sound either was continuous or contained a short gap in the middle. The target appeared with a high probability at the time point indicated by the cue and with a low probability at the other time point. For instance, when the cue predicted the short interval, the target was presented $600 \mathrm{~ms}$ (valid) after the cue on $67 \%$ of the trials and $1,200 \mathrm{~ms}$ (invalid) after the cue on $22 \%$ of the trials. In the remaining trials, no target appeared at all (catch trial). Participants were asked to respond as quickly and as accurately as possible to both valid and invalid targets and to press one button to targets with a gap and a second button to targets without a gap (choice reaction task).

We expected the participants to respond more quickly (and possibly also more accurately) to validly cued targets than to invalidly cued targets. If sensory-processing levels are affected by temporal orienting when attention is oriented to one time point, but not exclusively focused on it, the auditory $\mathrm{N} 1$ to validly cued targets should be larger than the N1 to invalidly cued targets. Because evidence for a modulation of later processes has been reported independently of which paradigm or stimulus modality was used (e.g., Griffin et al., 2002; Lange et al., 2003; Miniussi et al., 1999; Sanders \& Astheimer, 2008), we also expected an attention-related enhancement in a later positivity in the time range of the P3. 


\section{Cue}

(Non-) Target short

(Non-) Target long

(a)

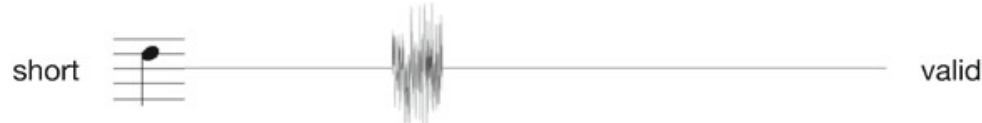

(b)

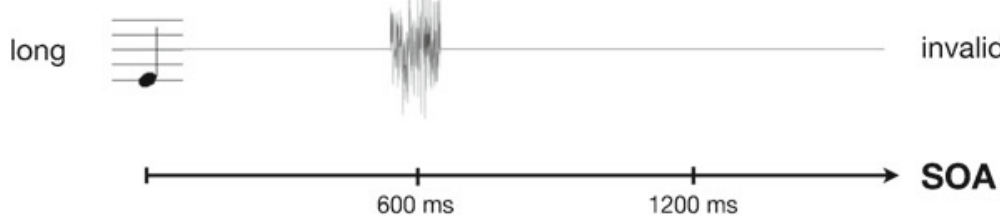

(c)

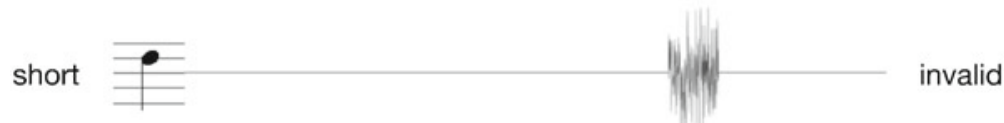

(d)

long

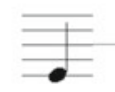

Fig. 1 Schematic illustration of the trial structure. Each trial contained a cue (high or low tone, indicated by the note symbol) that was followed after a short $(600-\mathrm{ms})$ or after a long $(1,200-\mathrm{ms})$ interval by a $50-\mathrm{ms}$ burst of white noise. The cue indicated when the noise burst was most likely to appear. For $50 \%$ of the participants, the high tone predicted the short interval, and the low tone the long interval; for

\section{Experiment 1}

\section{Method}

\section{Participants}

Twenty-eight healthy adults participated in the study. One participant was excluded from further analyses because of too many mistakes (more than one third of the responses were wrong). Three participants were excluded because of extensive artifacts in the electroencephalogram (EEG). The final sample comprised data of 24adults (15 female) 19-32 years of age (mean age, 23 years). Twenty of them were right-handed. All participants reported normal hearing. Visual acuity was normal or corrected to normal. The study was performed in accordance with the ethical standards laid down in the 1964 Declaration of Helsinki. All participants gave written informed consent and received course credit or a monetary compensation.

\section{Stimuli and task}

Each trial consisted of a cue and a target. The auditory cue was either a $391-\mathrm{Hz}$ (high tone) or a $261-\mathrm{Hz}$ (low tone) violin tone (duration, $100 \mathrm{~ms}$ ), generated with Reason 4 software. The target was a 100-ms white noise burst (generated with

$50 \%$, this assignment was reversed (not shown in the figure). The noise burst either was continuous or contained a short gap in the middle (not shown in the figure). Effects of temporal orienting were assessed by comparing behavioral responses and ERPs between valid and invalid trials for the short interval (a vs. b) and for the long interval (c vs. d)

Adobe Audition 1.5), which either was continuous or contained a 10-ms gap in the middle (gap). All auditory stimuli were presented binaurally via headphone (Koss 65), with a sound pressure level of $50 \mathrm{~dB}(\mathrm{~A})$.

The cue predicted the time point of target-onset, which could be 600 or 1,200 ms after cue-onset (i.e., the SOA was 600 or $1,200 \mathrm{~ms}$ for short and long intervals). The assignment of the high or low cue-tone to the short or the long timeinterval was counterbalanced across participants. The target appeared as predicted on $67 \%$ of the trials (valid trials). On $22 \%$ of the trials, the target appeared at the unpredicted point in time (invalid trials). On $11 \%$ of the trials, a target was presented at neither interval (catch trials). The participants were asked to press the right button with their right index finger to continuous targets and to press the left button with their left index finger to targets with a gap. The assignment of target to response side was counterbalanced across participants. A trial ended as soon as a response was given. If no response occurred, the trial ended $1,000 \mathrm{~ms}$ after the target tone. The intertrial interval varied randomly between 500 and 1,500 ms (rectangular distribution; mean, 1,000 ms).

To assess effects of temporal orienting, the processing of targets following short and long intervals were analyzed as a function of validity; that is, the processing of shortinterval targets when the short interval had been cued (valid 
short) was compared with the processing of short-interval targets when the long interval had been cued (invalid short). Likewise, the processing of long-interval targets when the long interval had been cued (valid long) was compared with the processing of long-interval targets when the short interval had been cued (invalid long; see also Fig. 1).

\section{Procedure}

Participants were seated in a dimly lit, electrically shielded room. Written instructions presented on the computer screen informed the participants about the relationship between cue and target and the types of responses they should perform. It was explicitly stated that the targets were more likely to appear after the short time interval following one kind of cue and after the long time interval following the other kind of cue. The participants were asked to respond to targets as quickly and as accurately as possible and to refrain from responding when no target appeared (i.e., on catchtrials). A small cross in the center of the screen should be focused during each block.

To clarify the relation between the cues and the intervals they predicted, the first practice block contained only trials with validly cued short intervals. The second practice block contained only validly cued long intervals. The first two practice blocks consisted of 18 trials each, 9 of which had a continuous target and 9 a gap target. The third practice block (36 trials) consisted of trials of all experimental conditions - that is, 12 valid short, 12 valid long, 4 invalid short, 4 invalid long, and 4 catch trials. During the practice blocks, participants received visual feedback after each trial.

The main experiment consisted of 20 blocks. Each block consisted of 72 trials: 64 with a target (24 valid short, 24 valid long, 8 invalid short, 8 invalid long) and 8 catch trials. Half of the targets were continuous; half contained a gap. Each block was followed by a short break that could be terminated by the participants. The participants received feedback about their performance (number of correct and incorrect responses and number of misses) at the end of each block. The whole testing session (including instructions, practice blocks, and application and removal of all electrodes) lasted about $2 \mathrm{~h}$.

\section{ERP recording}

The EEG was recorded from nine $\mathrm{Ag} / \mathrm{AgCl}$ electrodes, which were directly attached to the scalp at positions F3, Fz, F4, C3, Cz, C4, P3, Pz, and P4. The Electrooculogram was recorded using two electrodes at the outer canthi of the eyes (horizontal) and one electrode above and one below the left eye (vertical). All electrodes were referenced to the right earlobe. An additional electrode at the left earlobe served for offline rereferencing of the data to a linkedearlobereference. Impedances were kept below $5 \mathrm{k} \Omega$. A ground electrode was placed at the forehead above the nasion. The EEG was digitized at $250 \mathrm{~Hz}$, the band pass of the amplifiers (NuAmps, Neuroscan) was DC to $100 \mathrm{~Hz}$. Offline, data were filtered with a Butterworth Zero Phase filter (0.1-30 Hz, $24 \mathrm{~dB} /$ oct). The scalp electrode amplitudes were corrected for activity of the eye balls using the algorithm suggested by Gratton, Coles, and Donchin (1983). This algorithm corrects for ocular artifacts by generating a propagation factor that describes the relationship between the EEG and the EOG traces.

\section{Data analysis}

Behavioral data Trials were included in the analysis only if reaction times were within two standard deviations $(S D S)$ of the participant's mean reaction time in the corresponding condition ( $4.7 \%$ of the trials were rejected, on average). Effects of validity on reaction times and error rates were assessed with repeated measures analyses of variance (ANOVAs) with the factors interval (short vs. long) and validity (valid vs. invalid). For each participant, one third of the valid trials were randomly chosen to calculate the average reaction times, in order to account for the different probabilities of valid and invalid trials.

In most visual temporal-cuing studies, reaction time benefits are found only for the short interval (e.g., Coull \& Nobre, 1998; Miniussi et al., 1999). When no target is presented at the end of the short interval, the participants know that the target will be presented at the end of the long interval. It is thus conceivable that the participants reorient their attention to this time point, which may reduce the difference between cued and uncued conditions (Coull \& Nobre, 1998; Nobre, 2001). Therefore, analyses were also run separately for target at the end of the short interval and at the end of the long interval.

ERP data To measure processes of temporal orienting prior to the attended time point (e.g., Lange et al., 2003), ERPs to the cues were averaged separately as a function of the interval predicted by the cue (short or long) and the actual timing of the target (at the end of the short or long interval). Segments started $200 \mathrm{~ms}$ before cue onset and ended $1,800 \mathrm{~ms}$ after cue-onset (i.e., $600 \mathrm{~ms}$ after the end of the long interval). To measure effects of temporal orienting on target processing, ERPs to targets were averaged as a function of their timing (after the short vs. long interval) and validity (validly cued vs. invalidly cued). Segments started $200 \mathrm{~ms}$ before target onset and ended $600 \mathrm{~ms}$ after target onset. 
Both for the cue-related and for the target-related ERPs, segments were included into the average only if the correct response was given within $1,000 \mathrm{~ms}$ following the target. Moreover, segments were removed according to the following criteria. The maximal allowed voltage step per sampling point was $50 \mu \mathrm{V}$. The maximal allowed absolute difference of two values in the segment was $80 \mu \mathrm{V}$, and the lowest allowed activity for $100 \mathrm{~ms}$ was $0.05 \mu \mathrm{V}$. Participants' data were included in the analyses only if at least twothirds of the trials in each condition remained. As for the reaction time data, one third of the valid trials were chosen randomly to calculate the ERPs, to achieve a comparable signal-tonoise ratio for the ERPs in the valid and the invalid conditions.

Cue-related ERPs were referenced to a $200-\mathrm{ms}$ pre-cue baseline. Mean voltages were calculated for seven successive 40-ms epochs, starting $320 \mathrm{~ms}$ after the cue for the short interval and $920 \mathrm{~ms}$ after the cue for the long interval. To minimize misalignment of the waveforms based on the CNV-activity, the baseline was set from 0 to $50 \mathrm{~ms}$ relative to the target for target-related ERPs (Correa et al., 2006; Correa \& Nobre, 2008). On the basis of a visual inspection of the grand average waveforms, the N1 was quantified as the mean voltage in the interval between 100 and $140 \mathrm{~ms}$ after target-onset. The P3 was quantified as the mean voltage between 350 and $450 \mathrm{~ms}$ after target-onset.

For statistical analyses, electrodes F3, Fz, F4, C3, Cz, $\mathrm{C} 4, \mathrm{P} 3, \mathrm{Pz}$, and $\mathrm{P} 4$ were assigned one value of the factor anterior-central-posterior (ACP) and one level of the factor left-medial-right (LMR). Cue-related ERPs were analyzed using a repeated measures ANOVA involving the factors interval (short, long), time epoch (seven levels), validity (valid, invalid), LMR (three levels), and ACP (three levels). Target-ERPs were analyzed separately for the N1 and the P3 time epochs. The N1 was expected over the frontal and central scalp, and it was analyzed with a repeated measures ANOVA involving the factors interval (short, long), validity (valid, invalid), LMR (three levels), and ACP (frontal, central). The P3 was analyzed using a repeated measures ANOVA involving the factors interval (short, long), validity (valid, invalid), LMR (three levels), and ACP (three levels).

Appropriate hierarchical ANOVAs were calculated to analyze higher-order interactions (O'Brien \& Kaiser, 1985). For all analyses, the Huynh-Feldt correction was applied in order to compensate for violations of the sphericity assumption, where appropriate (Huynh \& Feldt, 1976). The corrected probabilities, together with the corresponding $\varepsilon$ values, are reported. As an effect size measure, partial $\eta^{2}$ is reported. All analyses were conducted using SAS 9.1. Only effects involving any of the experimental factors of interval or validity are reported.

\section{Results}

\section{Behavioral data}

Overall, participants responded more quickly to short- than to long-interval targets [main effect interval, $F(1,23)=12.25$, $p<.01]$. Although a significant effect of validity was not observed in the overall analysis, responses tended to be faster to targets at the cued time point than to targets at the uncued time point [main effect validity, $F(1,23)=$ $3.30, p=.08, \eta^{2}=.13$; see Table 1]. The interaction between interval and validity was not significant. However, because earlier cuing studies reported significant effects only for the short interval (e.g., Coull \& Nobre, 1998; Miniussi et al., 1999), separate analyses for short and long intervals were conducted. A validity effect was reliably recorded for the short interval: Participants responded significantly more quickly to valid than to invalid targets, $F(1,23)=8.00, p<.01, \eta^{2}=.26$. For the long interval, reaction times for valid and invalid targets did not differ significantly, $F(1,23)=0.72, p=.41, \eta^{2}=.03$.

Overall, participants were highly accurate in performing the task, as measured by the percentage of correct responses (see Table 1). Significant effects of interval or validity were not observed. However, the main effect of validity tended to be significant, $F(1,23)=3.02, p=.09, \eta^{2}=.12$. For the long interval, accuracy was actually lower for valid than for invalid targets, $F(1,23)=4.6, p=.04$, $\eta^{2}=.15$. For the short interval, the participants responded equally accurately to valid and invalid targets, $F(1,23)=0.07$, $p=.80, \eta^{2}=.00$.

\section{ERP data}

ERPs elicited by the cue were characterized by a pronounced N1-P2 complex, which was followed by a slow stimulus-preceding negativity (see Fig. 2). This negativity increased prior to the end of the short interval but decreased prior to the end of the long interval. Target-related ERPs were characterized by an N1 (peaking around $112 \mathrm{~ms}$ ) and a P3 (peaking around $400 \mathrm{~ms}$ ). The P3 amplitude (between 350 and $450 \mathrm{~ms}$ ) was enhanced for targets at the expected (valid) point in time, as compared with the unexpected (invalid) targets (see Fig. 3).

Cue-related ERPS An enhanced negativity developed between cue and target, with a maximum over the frontocentral scalp. Because the negativity had a different time course for the short and the long interval (interactions involving factors interval and time window; see Table 2), separate ANOVAs were run. For the short interval, the negativity increased over time (main effect time window; see Table 2), particularly over the left and right central and parietal scalp (time window*- 
Table 1 Mean reaction times and percentages correct for Experiment 1 (left panel) and mean reaction times, percentages of hits, and percentages of false alarms for Experiment 2 (right panel), separately for valid and invalid trials of short and long intervals. The standard errors of the meansare presented in parentheses

\begin{tabular}{|c|c|c|c|c|c|c|c|c|c|c|}
\hline & \multicolumn{4}{|c|}{ Experiment 1} & \multicolumn{6}{|c|}{ Experiment 2} \\
\hline & Short & & Long & & Short & & & Long & & \\
\hline & $\mathrm{RT}(S E)$ & $\%$ Correct $(S E)$ & $\mathrm{RT}(S E)$ & $\%$ Correct $(S E)$ & $\mathrm{RT}(S E)$ & $\%$ Hits $(S E)$ & $\%$ False Alarms $(S E)$ & RT $(S E)$ & $\%$ Hits $(S E)$ & $\%$ False Alarms $(S E)$ \\
\hline Valid & 467 (11) & $96.63(0.49)$ & $478(11)$ & $96.75(0.63)$ & $404(14)$ & $99.53(0.15)$ & $0.93(0.23)$ & 419 (13) & $99.73(0.14)$ & $0.73(0.21)$ \\
\hline Invalid & $476(11)$ & $96.72(0.52)$ & $483(12)$ & $97.42(0.55)$ & 412 (14) & $99.73(0.21)$ & $1.27(0.31)$ & $420(14)$ & $99.80(0.11)$ & $0.67(0.25)$ \\
\hline
\end{tabular}

ACP*LMR; see Table 2). For the long interval, the lasting negativity slightly decreased, particularly over the right and central scalp (time window*ACP; see Table 2). Notably, effects of temporal orienting were not observed in the slow negativity following the cue (effects involving the factor validity: all $p \mathrm{~s}>.12$ ).

Target ERPs: N1 time range $(100-140 \mathrm{~ms})$ For the N1 time range, effects of validity were differently pronounced for short and long intervals [interval*validity, $F(1,23)=5.92$, $p=.02, \eta^{2}=.20$; interval*validity*ACP*LMR, $F(2,46)=$ $\left.4.07, p=.03, \varepsilon=0.79, \eta^{2}=.15\right]$. This was due to the fact that mean amplitudes of the N1 did not differ significantly between valid and invalid targets for the short interval (all $p \mathrm{~s}>.14$ ), whereas the N1 to valid targets was smaller than the N1 to invalid targets for the long interval over the central scalp [validity*ACP, $F(1,23)=4.4, p<.05, \eta^{2}=.16$ ].
Target ERPs: P3 time range (350-450 ms) Amplitudes between 300 and $450 \mathrm{~ms}$ were more positive for valid than for invalid targets [validity*ACP, $F(2,46)=8.07, p<.01, \varepsilon=$ $\left.0.73, \eta^{2}=.26\right]$. The main effect of validity was not significant for single electrode clusters, but it was most pronounced over the parietal and central scalp. Interactions involving both validity and interval were not observed (all $p s>.26$ ), and the effect of validity was reliable both for the short and for the long intervals [short, validity*ACP, $F(2,46)=3.83, p=.04$, $\varepsilon=0.75, \eta^{2}=.14$; long,validity*ACP, $F(2,46)=6.74, p<.01$, $\left.\varepsilon=0.65, \eta^{2}=.23\right]$.

\section{Discussion}

One reason to conduct Experiment 1 was to provide evidence for the functional validity of auditory temporal
Fig. 2 Experiment 1: Grand average ERPs $(\mathrm{Cz})$ elicited by cues that indicated the target onset after the short interval (thick lines) and after the long interval (thin lines), validly (solid lines) and invalidly (dashed lines). ERPs are referred to a $200-\mathrm{ms}$ pre-cue baseline. The onsets of the cue and of the short and long interval targets are indicated by vertical lines at 0,600 , and 1,200 ms. N1 (100$140 \mathrm{~ms})$ and P3 (350-450 ms) time ranges are highlighted in gray. In all panels, negativity is up

\section{Cue-related ERPs Experiment 1}

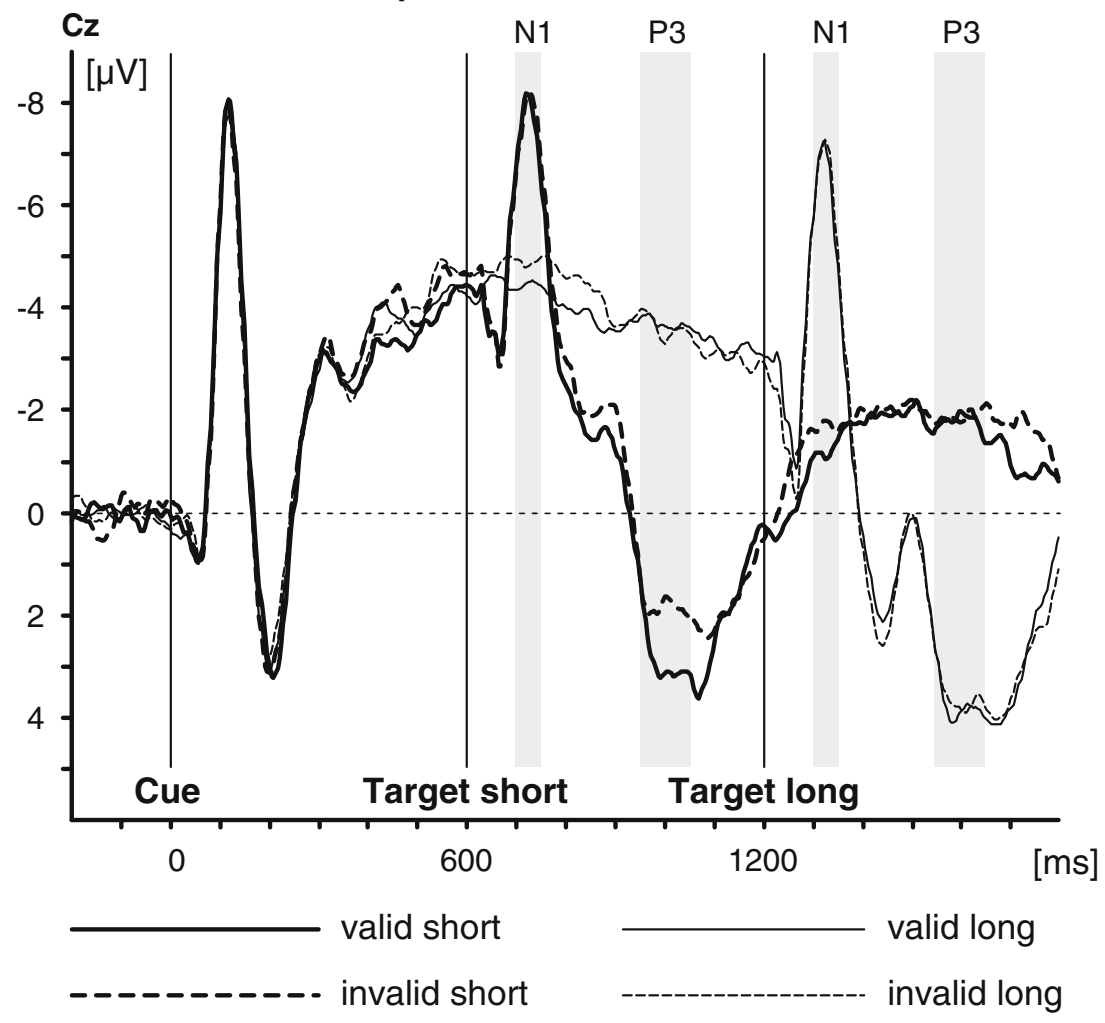




\section{Experiment 1}

\section{ERPs of the short interval targets}

C3
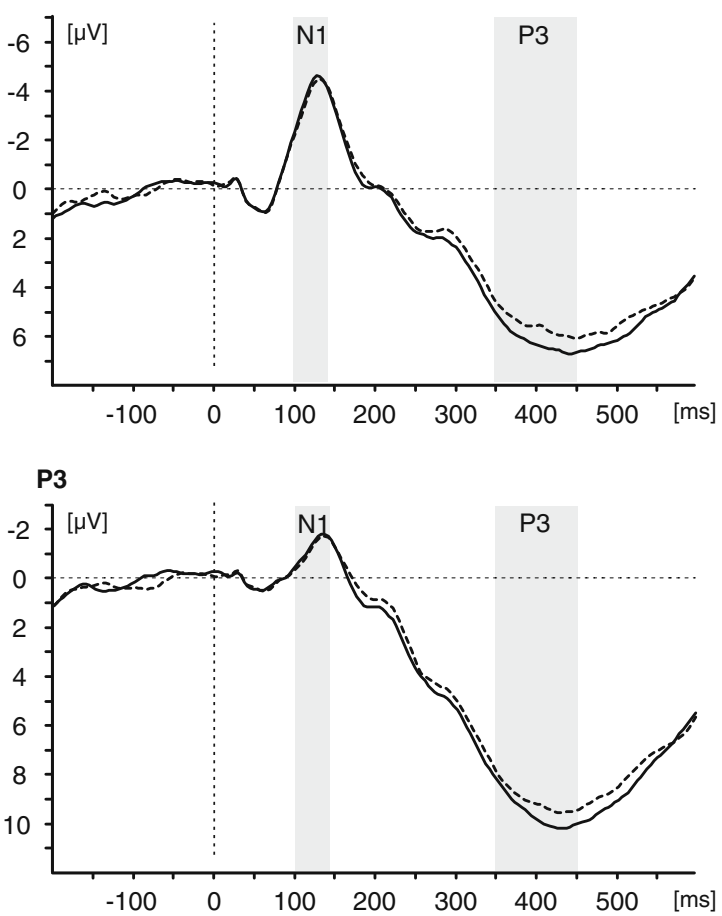

\section{ERPs of the long interval targets}
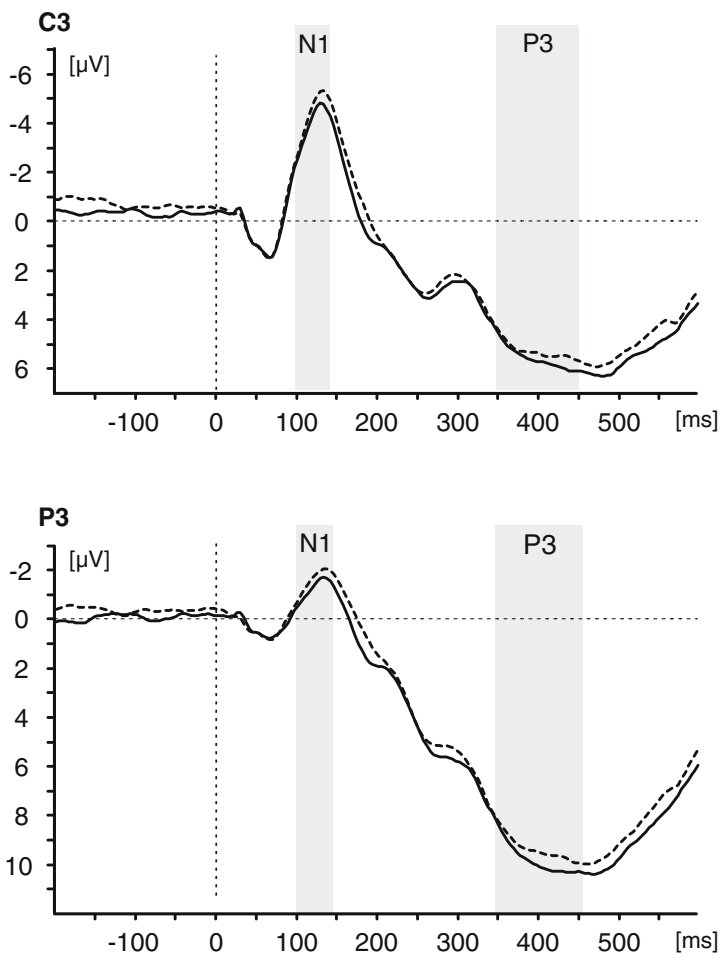

Fig. 3 Experiment 1: Grand average ERPs elicited by valid (solid lines) and invalid (dashed lines) targets at the end of the short interval (top) and at the end of the long interval (bottom), for electrodes $\mathrm{C} 3$,
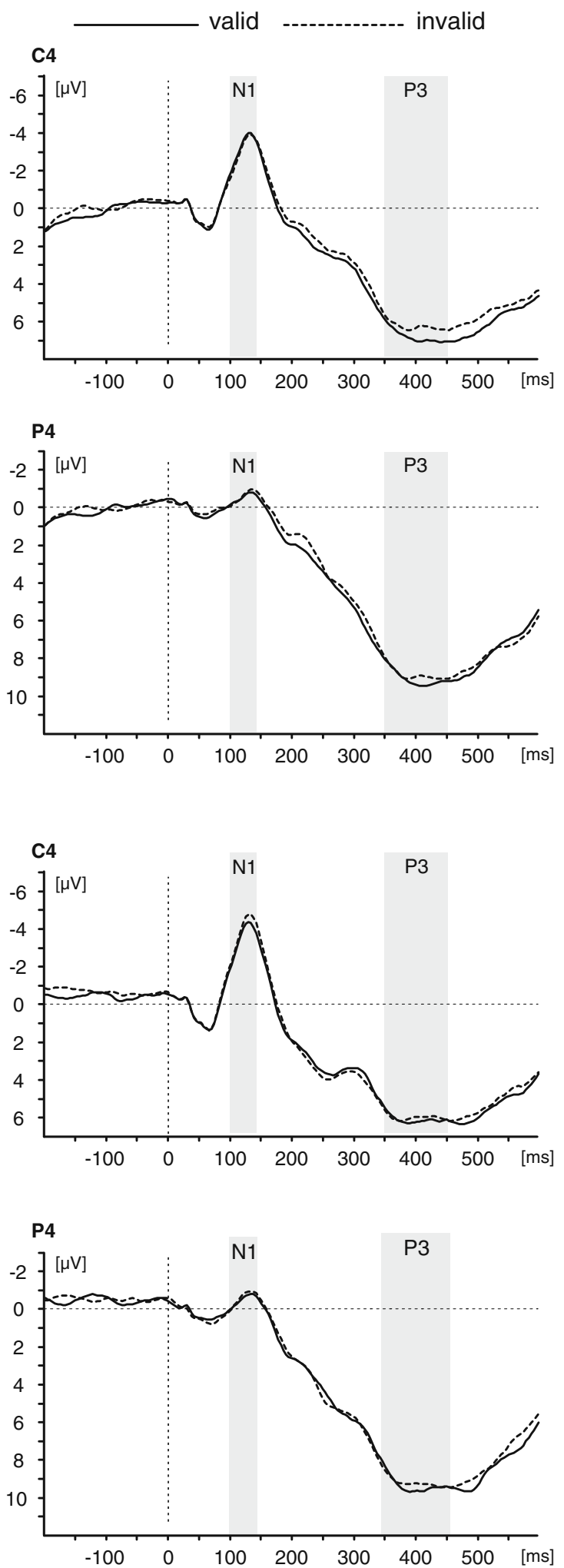

$\mathrm{C} 4, \mathrm{P} 3$, and P4. ERPs are referred to a 50-ms-long poststimulus baseline. Target-onset is indicated by a dashed vertical line. N1 (100 $140 \mathrm{~ms}$ ) and P3 (350-450 ms) time ranges are highlighted in gray 
Table 2 Experiment 1:

Analysis of cue-related ERPs: ANOVA on mean amplitudes for seven successive 40-ms time windows, starting $280 \mathrm{~ms}$ prior to the target. Only significant effects involving the factors validity or interval are displayed

\begin{tabular}{|c|c|c|c|c|c|}
\hline Effect & $\mathrm{df}$ & $\mathrm{F}$ & $\mathrm{p}$ & $\varepsilon$ & $\eta^{2}$ \\
\hline \multicolumn{6}{|l|}{ Experiment 1} \\
\hline Interval*ACP & $12 / 276$ & 2.46 & .03 & 0.73 & .10 \\
\hline Interval*ACP*LMR & $24 / 552$ & 4.92 & .02 & 0.92 & .18 \\
\hline Time window*interval & $6 / 138$ & 11.05 & $<.01$ & 0.36 & .32 \\
\hline Time window*interval*ACP & $12 / 276$ & 4.66 & $<.01$ & 0.33 & .17 \\
\hline Time window*interval*ACP*LMR & $24 / 552$ & 3.22 & $<.01$ & 0.35 & .12 \\
\hline \multicolumn{6}{|l|}{ Experiment 2} \\
\hline Interval*ACP & $2 / 48$ & 23.80 & $<.01$ & 0.75 & .50 \\
\hline Interval*ACP*LMR & $4 / 96$ & 7.70 & $<.01$ & 1.02 & .24 \\
\hline Interval*ACP*time window & $12 / 288$ & 77.75 & $<.01$ & 0.48 & .76 \\
\hline Validity & $1 / 24$ & 18.14 & $<.01$ & - & .43 \\
\hline Validity*ACP & $2 / 48$ & 5.73 & .01 & 0.73 & .19 \\
\hline Validity*ACP*LMR & $4 / 96$ & 3.14 & .03 & 0.78 & .12 \\
\hline Validity*time window & $6 / 144$ & 5.67 & $<.01$ & 0.73 & .19 \\
\hline Interval*validity*ACP*LMR & $4 / 96$ & 5.9 & $<.01$ & 0.87 & .20 \\
\hline Interval*validity*time window & $6 / 144$ & 2.45 & $<.05$ & 0.72 & .09 \\
\hline \multicolumn{6}{|l|}{ Short Interval } \\
\hline Validity & $1 / 24$ & 7.46 & .01 & - & .24 \\
\hline Validity*ACP & $2 / 48$ & 6.62 & $<.01$ & 0.75 & .22 \\
\hline \multicolumn{6}{|l|}{ Long Interval } \\
\hline Validity & $1 / 24$ & 13.06 & .01 & - & .35 \\
\hline Validity*ACP & $2 / 48$ & 4.24 & $<.03$ & 0.84 & .15 \\
\hline Validity*ACP*LMR & $4 / 96$ & 6.53 & $<.01$ & 0.75 & .21 \\
\hline Validity*time window & $6 / 144$ & 8.17 & $<.01$ & 0.68 & .25 \\
\hline Validity*time window*ACP & $12 / 288$ & 2.52 & $<.05$ & 0.33 & .10 \\
\hline
\end{tabular}

orienting by demonstrating that it improves stimulus processing at a behavioral level. For the short (but not the long) interval, the participants responded more quickly to valid targets than to invalid targets. Because accuracy was not affected, the reaction time benefit was not simply due to a speed-accuracy trade-off. Thus, by showing a behavioral benefit for valid targets in an auditory temporal-cuing task, Experiment 1 provides evidence for the functional significance of temporal orienting in the auditory modality. Consistent with findings of the visual-cuing studies (e.g., Coull \& Nobre, 1998; Griffin et al., 2002; Miniussi et al., 1999), this effect was restricted to the short interval, which may be due to a reorienting of attention to the long interval (e.g., Coull \& Nobre, 1998).

The second goal of Experiment 1 was to investigate the effects of auditory temporal orienting when attention is not selectively oriented to a time point but, rather, divided between a cued and an uncued moment: Is it based on a modulation of early sensory processing, or does temporal orienting increase the efficiency of processes related to stimulus evaluation or response selection? The ERP data of Experiment 1 support the latter view: For the short interval, where behavioral data provided evidence of temporal orienting, an enhancement of a parietal positivity in the time range of the P3 was found, but no effect in the auditory N1.
This finding is in line with the results of visual temporal-cuing studies (e.g., Griffin et al., 2002, Experiment 2; Miniussi et al., 1999) and, thus, supports the notion that temporal orienting improves stimulus processing mainly by modulating decision- or motor-related processes (Nobre, 2001).

A slow negativity developed between cue and target of both short and long intervals over the frontocentral scalp. This slow potential shift might belong to the CNV family (e.g., Walter, Winter, Cooper, McCallum, \& Aldridge, 1964). Among the processes that have been discussed to contribute to the $\mathrm{CNV}$ are the anticipation of a stimulus (e.g., Walter et al., 1964), the timing of the interval (e.g., Elbert, Ulrich, Rockstroh, \& Lutzenberger, 1991; Macar \& Besson, 1985; McAdam, 1967; Pouthas, Garnero, Ferrandez, \& Renault, 2000; Ruchkin, McCalley, \& Glaser, 1977; for a review, see Pouthas, 2003), and the preparation of a (motor) response (e.g., Birbaumer, Elbert, Canavan, \& Rockstroh, 1990). Similar potential drifts following the cue have been reported in earlier temporal-orienting studies (Correa et al., 2006; Griffin et al., 2002; Lange et al., 2003; Miniussi et al., 1999; Sanders \& Astheimer, 2008). Notably, these earlier studies showed larger negativities prior to the attended time point, suggesting a role in attentional control (e.g., Correa et al., 2006; Lange et al., 2003). By contrast to 
these earlier studies, signs of temporal orienting were not observed in the slow waves in Experiment 1. However, the valid/invalid ratio was smaller in the present study $(67 \%$ valid trials, $22 \%$ invalid trials) than in earlier temporalcuing studies (e.g., in the study of Miniussi and colleagues: $80 \%$ valid trials, $10 \%$ invalid trials). It may be hypothesized that this led to a less pronounced bias toward the valid time point in the present study than in earlier ones. Nevertheless, a reaction time benefit was observed in the present study. This finding supports the notion that the difference between the proportions of valid and invalid trials was large enough to induce an attentional bias for the cued time point.

Unexpectedly, more accurate processing of uncued targets and a larger N1 to uncued targets were observed for targets of the long interval. This is at odds with the notion that auditory temporal orienting improves stimulus processing. Together with the larger positivity to cued longinterval targets, this pattern is inconsistent with the findings of earlier visual-cuing studies using two intervals, where no effects of temporal orienting were observed for the long interval (Coull \& Nobre, 1998; Griffin et al., 2002; Miniussi et al., 1999; but see Correa et al., 2006). Before discussing these unpredicted results, we will first test the reliability of this pattern in a second experiment.

\section{Experiment 2}

For the short interval, the pattern of results observed in Experiment 1 was similar to what was reported by the visual-cuing studies: Participants responded more quickly to valid than to invalid targets. This behavioral effect was accompanied by an enhanced P3, but not by earlier modulations in the auditory N1. Thus, modulations at sensory-processing levels are not observed in the auditory modality when attention is divided between a cued and an uncued moment. This is in line with the notion that temporal orienting improves stimulus processing mainly by modulating decision- or motor-related processes (Nobre, 2001). Yet there is evidence that temporal orienting can affect sensory stages of auditory processing: Studies using an experimental paradigm that allows for a selective orienting of attention to a particular time point consistently reported an enhancement of the auditory $\mathrm{N} 1$ - in addition to a later attention-related positivity in the time range of the P3 (e.g., Lange et al., 2006; Lange \& Röder, 2006; Lange et al., 2003; Röder et al., 2007; Sanders \& Astheimer, 2008; for a review, see Lange \& Röder, 2010). The selective temporal-orienting paradigm used in these studies differed from the cuing task of Experiment 1 in several ways, each of which might contribute to a modulation of stimulus processing at early perceptual levels.
First, attention could be selectively focused on the attended time point in the selective temporal-orienting paradigm, because only stimuli at the attended time point were relevant for response-selection. By contrast, in the temporal-cuing paradigm of Experiment 1, attention had to be divided between cued and uncued time points, because targets required a response irrespective of the time point indicated by the cue. The processing stage affected by temporal orienting may thus be influenced by the degree to which attention is focused around a particular time point (see Eimer, 1994, 1996, for a similar idea in spatial orienting). Moreover, in the selective temporal-orienting paradigm, attention is directed to the same time point for an entire block (i.e., the focus of attention varies between blocks). In the cuing task, by contrast, attention is oriented in a trial-by-trial manner (i.e., the focus of attention varies within blocks). Notably, Correa and colleagues found temporal-orienting effects in vision to be larger when attention was manipulated between blocks, rather than within blocks (Correa et al., 2004). When attention was manipulated between blocks, these authors also observed an enhancement of the sensory evoked P1 in a visual temporal-cuing task (Correa et al., 2006). It has been suggested that it is easier to generate a precise representation of the to-be-attended time interval with a constant focus of attention, ascompared with a condition in which the to-beattended point changes every trial (Correa et al., 2006; Lange \& Röder, 2006). This may facilitate the discrimination between attended and unattended intervals and may,thus, favor a particularly early selection.

The goal of Experiment 2 was to assess whether temporal orienting enhances early, sensory levels of auditory processing when attention is manipulated in a trial-by-trial manner (as in Experiment 1) but can be focused selectively on the cued time point (as in the selective temporal-orienting paradigm; e.g., Lange et al., 2003; Sanders \& Astheimer, 2008). Thus, the trial-by-trial cuing task of Experiment 1 was slightly adapted. Participants were asked to respond only to stimuli at the time point indicated by the cue. Stimuli at the uncued time point were no longer relevant for response selection. Therefore, this task should give rise to a selective orienting of attention to the time point indicated by the cue. To still be able to compare ERPs elicited in physically identical conditions (i.e., to physically identical stimuli not associated with a response), we used a go/no-go task instead of the choice reaction task employed in Experiment 1. Participants were asked to respond only to a small subset of the validly cued stimuli (target), but not to frequent (nontarget) stimuli. Thus, neither for the cued nor for the uncued time pointwere nontargets associated with a response. Effects of selective temporal orienting on auditory processing were assessed by comparing ERPs to physically identical nontargets under physically identical conditions (no response required). To assess the validity of the go/no-go 
cuing paradigm on a behavioral level, each participant also took part in a behavioral session, where both the cued and the uncued time points were relevant for response selection.

For the behavioral session, we expected the participants to respond more quickly to validly cued targets (of the short interval) than to invalidly cued targets, as in Experiment 1. If sensory-processing levels are affected when attention is selectively oriented to a particular time point in a trial-by-trial manner, the auditory N1 to validly cued nontargets should be larger than the N1 to invalidly cued nontargets. Selective temporal orienting should also affect later processes, as indicated in the late positivity.

\section{Method}

\section{Participants}

Twenty-seven healthy adults participated in the study. Data of 2 participants were excluded from further analyses because of extensive artifacts in the EEG recordings. The final sample consisted of 25 participants (17 female; 1938 years of age; mean age, 25 years). Nineteen were righthanded. All participants reported normal hearing. Visual acuity was normal or corrected to normal. The study was performed in accordance with the ethical standards laid down in the 1964 Declaration of Helsinki. All participants gave written informed consent and received course credit or a monetary compensation.

\section{Procedure}

Experiment 2 was identical to Experiment 1, except for the following. Most important, only stimuli that appeared at the point in time predicted by the cue (i.e., valid targets) were relevant for response selection. Invalid stimuli could be completely ignored. A go/no-go task was used. Participants were asked to press a button with their right index finger if a validly cued stimulus contained a gap (target) and to refrain from responding if the valid stimulus was continuous (nontarget). Invalid stimuli required no reaction at all. Target and nontarget stimuli were presented randomly to the left or to the right ear. Only the cues were presented binaurally, as in Experiment 1.

Because participants were asked to respond only to valid targets, effects of temporal orienting could not be examined in the behavioral data obtained in the EEG experiment. To test whether temporal orienting improves auditory processing also when a go/no-go task is used, participants were tested in an additional session with a behavioral version of the go/nogo task, in which both valid and invalid targets required a response. The behavioral session was always conducted before the EEG session so that the participants were familiar with the basic structure of the experiment before starting the
EEG session. The participants were asked to press a button with their right index finger when the stimulus contained a gap (target) and to refrain from responding when the stimulus was continuous (nontarget), regardless of validity. The participants completed 2 practice blocks with only valid short and valid long trials, respectively ( 8 trials with a continuous stimulus as target, 8 trials with a gapstimulus) and a third practice block that included all possible conditions of the experiment ( 8 valid continuous, 8 valid gap, 4 invalid continuous, 4 invalid gap, 2 catch trials). The entire behavioral experiment consisted of 10 blocks with 78 trials each ( 24 valid continuous, 24 valid gap, 12 invalid continuous, 12 invalid gap, and 6 catch trials). Of the trials that ended with a stimulus, the interval was short on half of the trials and long on the other half. Thus, each behavioral block contained about $62 \%$ valid trials, $31 \%$ invalid trials, and $8 \%$ catchtrials. Breaks and feedback were the same as in Experiment 1. The behavioral session lasted about $1 \mathrm{~h}$. Attention effects were assessed by comparing reaction times to valid and invalid targets and false alarms to valid and invalid nontargets. Trials were included in the analysis only if reaction times were within two $S D$ s of the participant's mean reaction time in the corresponding condition $(4.6 \%$ of the trials were rejected, on average). Effects of validity on reaction times, percentages of hits, and percentages of false alarms were analyzed as a function of interval (short, long) and validity (valid, invalid).

In the EEG session, each participant was again familiarized with the task during three practice blocks. As in the behavioral session, the participants fulfilled 2 practice blocks with only valid short and valid long trials, respectively ( 8 trials with a continuous stimulus as target, 8 trials with a gapstimulus). The third practice block contained 38 trials (16 valid with a continuous stimulus, 8 valid with a gapstimulus, 8 invalid with a continuous stimulus, 4 invalid with a gapstimulus, and 2 catch trials). Of the trials with a stimulus, the interval was short on half of the trials and long on the other half. The main experiment consisted of 20 blocks with 76 trials each (32 valid continuous, 16 valid gap, 16 invalid continuous, 8 invalid gap, and 4 catch trials). Thus, each block contained $63 \%$ valid trials, 32\% invalid trials, and 5\% catchtrials. Attention effects were assessed by comparing ERPs to valid and invalid nontargets, which were physically identical and were not associated with a response. Breaks and feedback were the same as in Experiment 1. The whole EEG session lasted about $2 \mathrm{~h}$.

\section{ERP recording and analysis}

The EEG was recorded from 29 active electrodes (ActiCaps; Brain Products, Gilching, Germany), mounted in an elastic cap (F7, F3, Fz, F4, F8, FC5, FC1, FCz, FC2, FC6, T7, C3, $\mathrm{Cz}, \mathrm{C} 4, \mathrm{~T} 8, \mathrm{CP} 5, \mathrm{CP} 1, \mathrm{CP} 2, \mathrm{CP} 6, \mathrm{P} 7, \mathrm{P} 3, \mathrm{Pz}, \mathrm{P} 4, \mathrm{P} 8, \mathrm{O} 1, \mathrm{Oz}$, $\mathrm{O} 2$, PO9, PO10). Horizontal eye movements were monitored by measuring the difference of electrodes F7 and F8. Vertical 
eye movements were measured by electrode FP2. During recording, all electrodes were referenced to $\mathrm{FCz}$. A ground electrode was placed at position AFc. All electrode impedances were kept at $25 \mathrm{k} \Omega$ or below. Offline, all the electrodes were rereferenced to linked mastoids. The EEG was filtered by a Butterworth Zero Phase Filter with a low cut-off at $0.1 \mathrm{~Hz}$ and a high cut-off at $30 \mathrm{~Hz}, 24 \mathrm{~dB} /$ oct. The scalp electrode amplitudes were corrected for ocular activity (Gratton et al., 1983).

To measure processes of temporal orienting prior to the attended time point, cue-related ERPs were averaged separately as a function of the interval predicted by the cue (short or long) and the actual timing of the nontarget stimulus (at the end of the short or long interval). Trials were included into the average only if a nontarget stimulus occurred and if no response was given within the $1,000 \mathrm{~ms}$ following this nontarget,as requested by the task. ERPs to validly and invalidly cued nontargets were averaged separately for the short and for the long intervals, if no response was given within the $1,000 \mathrm{~ms}$ following the stimulus. Segments started $200 \mathrm{~ms}$ before target onset and ended $600 \mathrm{~ms}$ thereafter. As in Experiment 1, the baseline was set from 0 to $50 \mathrm{~ms}$ to avoid a misalignment of the N1 wave by CNV activity. On the basis of visual inspection of the grand average waveforms, N1 amplitudes were quantified as the mean voltage in the interval between 90 and $130 \mathrm{~ms}$ after onset of the nontarget. P3 amplitudes were quantified as the mean voltage between 250 and $400 \mathrm{~ms}$ after onset of the nontarget. Statistical analyses were conducted in the same way as in Experiment 1. Only effects involving any of the experimental factors of interval or validity are reported. Segmentation, artifact rejection, and statistical analyses were identical to those in Experiment 1.

\section{Results}

\section{Behavioral experiment}

Overall, participants responded more quickly to targets following a short than following a long interval [main effect interval, $\left.F(1,24)=9.83, p<.01, \eta^{2}=.29\right]$ and more quickly to valid than to invalid targets [main effect validity, $F(1,24)=4.38, p<.05, \eta^{2}=.15$; see Table 1 for the descriptive values]. The interaction was not significant. Because earlier studies, as well as Experiment 1, observed a validity effect only for the short interval, separate analyses were conducted for short and long intervals. When the interval was short, participants responded more quickly to valid than to invalid targets, $F(1,24)=4.62, p=.04, \eta^{2}=.16$. For the long interval, reaction times for valid and invalid targets did not differ significantly, $F<1$.

Participants' responding was highly accurate: They responded to more than $99 \%$ of the targets, but to only about $1 \%$ of the nontargets (see Table 1 ). An effect of trial validity was observed neither for the hits nor for the false alarms $(p>.22)$. Separate analyses for the short and the long intervals confirmed this pattern (all $p$ s $>.22$ ). However, both the hits and the false alarms showed that participants tended to respond more accurately if the interval was long than when the interval was short [main effect interval: hits, $F(1,24)=3.97, p=.06, \eta^{2}=.14$; false alarms, $\left.F(1,24)=3.67, p=.07, \eta^{2}=.13\right]$.

\section{ERP data}

Cue-related ERPs were characterized by a pronounced N1-P2 complex, which was followed by a slow stimuluspreceding negativity over the frontocentral scalp. Between 320 and $600 \mathrm{~ms}$ (i.e., prior to the end of the short interval), this negative slow wave was larger when the cue had indicated the short interval. By contrast, between 920 and $1,200 \mathrm{~ms}$ (i.e., prior to the end of the long interval), negativity was more pronounced when the cue had indicated the long interval (see Fig. 4). ERPs to nontargets were characterized by an N1 peaking around $112 \mathrm{~ms}$ and a P3 peaking around $350 \mathrm{~ms}$. For the short interval, valid nontargets elicited a larger N1 $(90-130 \mathrm{~ms})$ than did invalid nontargets over the left scalp. For the long interval, negativity was larger for invalid than for valid nontargets. For both intervals, temporal orienting was associated with an enhancement of the P3 (250-400 ms; see Fig. 5).

Cue-related ERPS The overall ANOVA revealed several higher-order interactions involving the factors time window, interval, validity, ACP, and LMR (see Table 2), indicating a long-lasting negativity, which was larger prior to the cued time point. Prior to the end of the short interval, negativity was larger if the cue indicated the short interval, particularly over frontal electrodes (effects involving the factor validity for the short interval; see Table 2). The validity effect did, however, not vary significantly over time. Prior to the end of the long interval, negativity was larger if the long interval had been indicated by the cue, particularly over the central scalp (effects involving factor validity for the long interval; see Table 2). This effect became larger over time (effects involving factor validity and time window for the long interval; see Table 2).

ERPs to nontargets: The N1 $(90-130 \mathrm{~ms})$ The overall ANOVA showed that effects of validity in the N1 time range were different for nontargets of short and long intervals [interval*validity, $F(1,24)=7.38, p=.01$, $\eta^{2}=.24 ;$ interval ${ }^{*}$ validity*ACP, $F(1,24)=6.25, p=.02$, $\left.\eta^{2}=.21\right]$. Sub-ANOVAs conducted for the short interval revealed that the N1 was larger to valid nontargets than to invalid nontargets [validity*LMR, $F(2,48)=3.58, p=.04$; 
Fig. 4 Experiment 2: Grand average ERPs $(\mathrm{Cz})$ elicited by cues that indicated the onset of the target or nontarget after the short interval (thick lines) and after the long interval (thin lines), validly (solid lines) and invalidly (dashed lines). ERPs are referred to a 200-ms-long pre-cue baseline. The onsets of the cue and of the short- and long-interval targets or nontargets are indicated by vertical lines at 0,600 , and $1,200 \mathrm{~ms}$. $\mathrm{N} 1$ (90-130 ms) and P3 (250$400 \mathrm{~ms}$ ) time ranges are highlighted in gray

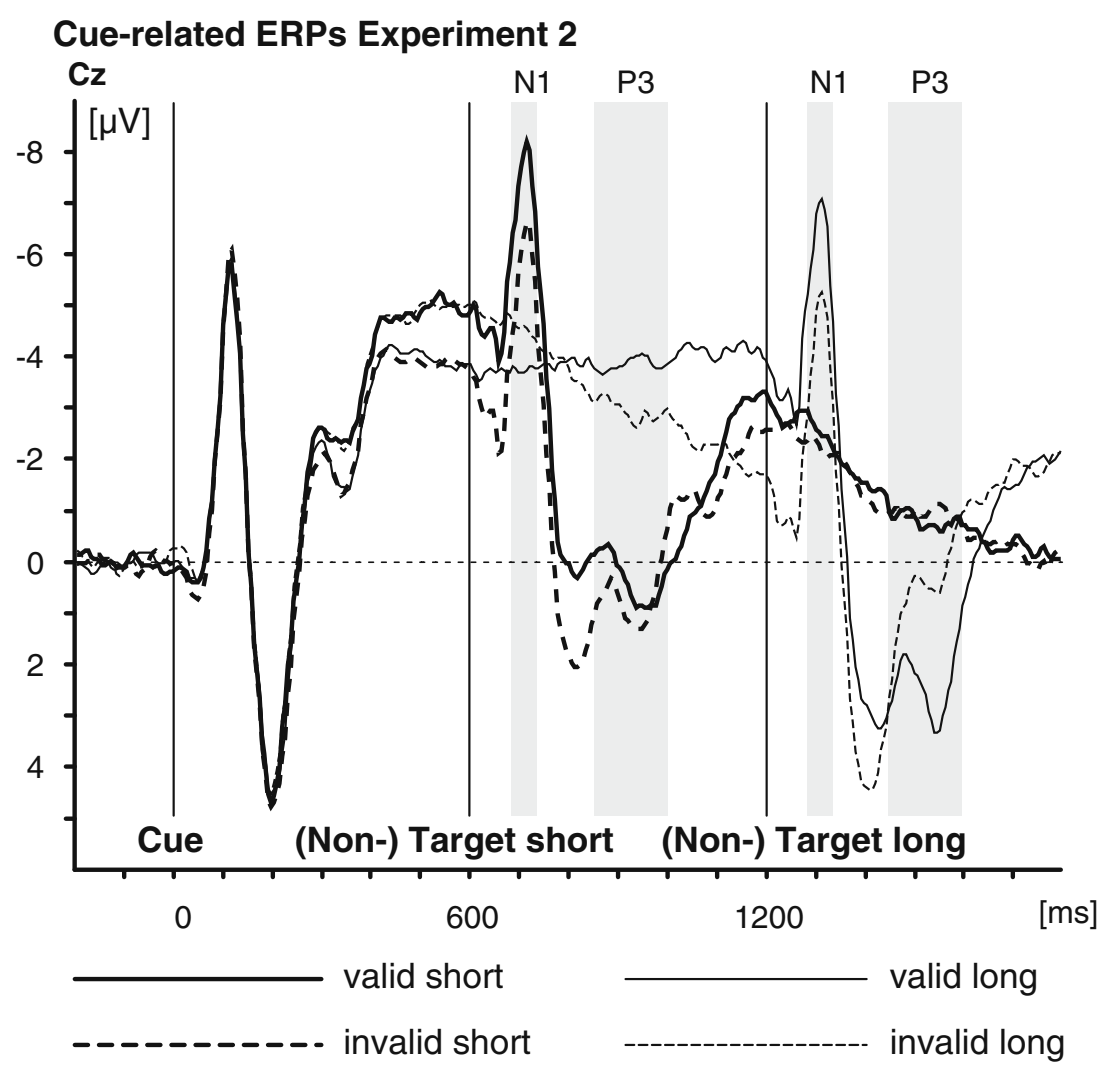

$\left.\varepsilon=0.98, \eta^{2}=.13\right]$. Separate analyses for left, medial, and right electrodes showed that this effect was significant only for the left hemisphere, $F(1,24)=7.45, p=.01, \eta^{2}=.24$, but neither for medial $(p>.29)$ nor for right electrodes, $F<1$. Further analyses for the long interval showed that the N1 was smaller to valid than to invalid nontargets [validity, $F(1,24)=5.12, p=.03, \eta^{2}=.18$; validity*ACP, $F(1,24)=$ $\left.4.32, p<.05, \eta^{2}=.15\right]$. Separate analyses for the frontal and the central electrodes showed that this effect was significant only for central, $F(1,24)=6.44, p=.02, \eta^{2}=.21$, but not for frontal $(p>.10)$ scalp sites.

Topographic comparison of the validity effect preceding the stimulus to the N1 validity effect To additionally test whether the validity effects observed in the N1 could be due to a continuation of the slow negativity preceding the end of the interval, rather than a modulation of processing stages associated with the nontarget proper, the difference potentials [ERP(valid) - ERP(invalid)] for the N1 (90$130 \mathrm{~ms}$ ) of the ERP to nontargets and for the 40-ms cuerelated ERP that preceded the end of the interval (560-600 and 1,160-1,200 ms for short and long intervals) were calculated separately for long and short intervals. These scores were submitted to a repeated measures ANOVA with the factors interval (short vs. long), pre-post (before or after the end of the interval), LMR (three levels), and ACP (three levels). Analyses were conducted both on the raw and on the normalized difference values (normalized separately for each level of interval, pre-post, and participant $(M=5, S D=2$; McCarthy \& Wood, 1985; Urbach \& Kutas, 2002).

Significant interactions of factors pre-post and interval with the topographic factors were observed (see Table 3), suggesting that the validity effect observed for the N1 was not a residual of the validity effect already present prior to the presentation of the nontarget stimulus. This was the case for both the short and the long intervals.

P3 time range (250-400 $\mathrm{ms})$ Valid nontargets were associated with a larger P3 than were invalid nontargets, particularly over the central and parietal scalp, which was differently pronounced for the short and the long intervals (see effects involving validity and interval in Table 4). A larger P3 for valid than for invalid nontargets was observed for both the short and long intervals. For the short interval, the P3 enhancement was restricted to the central scalp. For the long interval, the effect had a centro-parietal maximum but was reliable over the frontal scalp, as well (see Table 4).

Fig. 5 Experiment 2: Grand average ERPs elicited by valid (solid lines) and invalid (dashed lines) nontargets at the end of the short interval (top pattern) and at the end of the long interval (bottom pattern), for electrodes C3, C4, P3, and P4. ERPs are referred to a 50ms-long poststimulus baseline. The onset of the nontarget is indicated by a dashed vertical line. N1 (90-130 ms) and P3 (250-400 ms) time ranges are highlighted in gray 


\section{Experiment 2}

ERPs of the short interval non-targets

\section{C3}
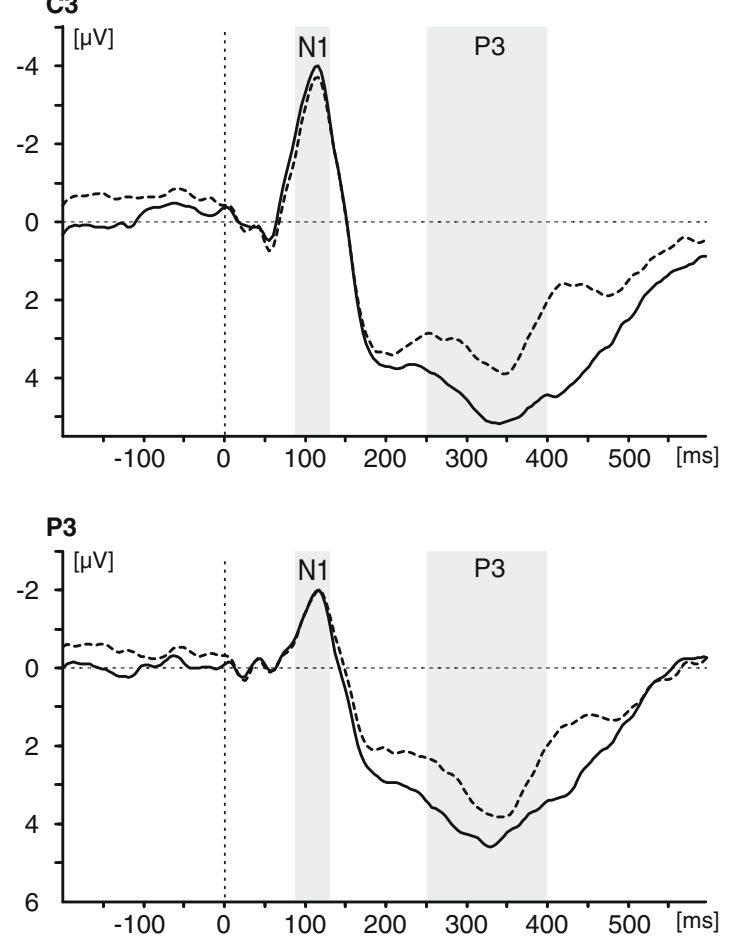

ERPs of the long interval non-targets
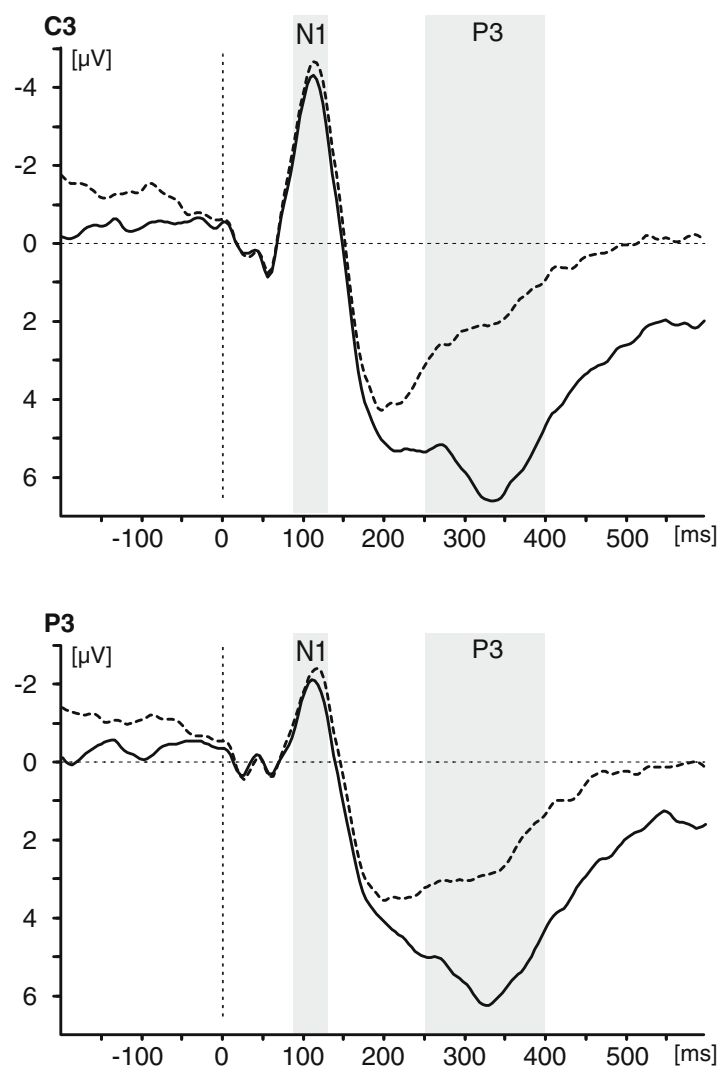

valid invalid
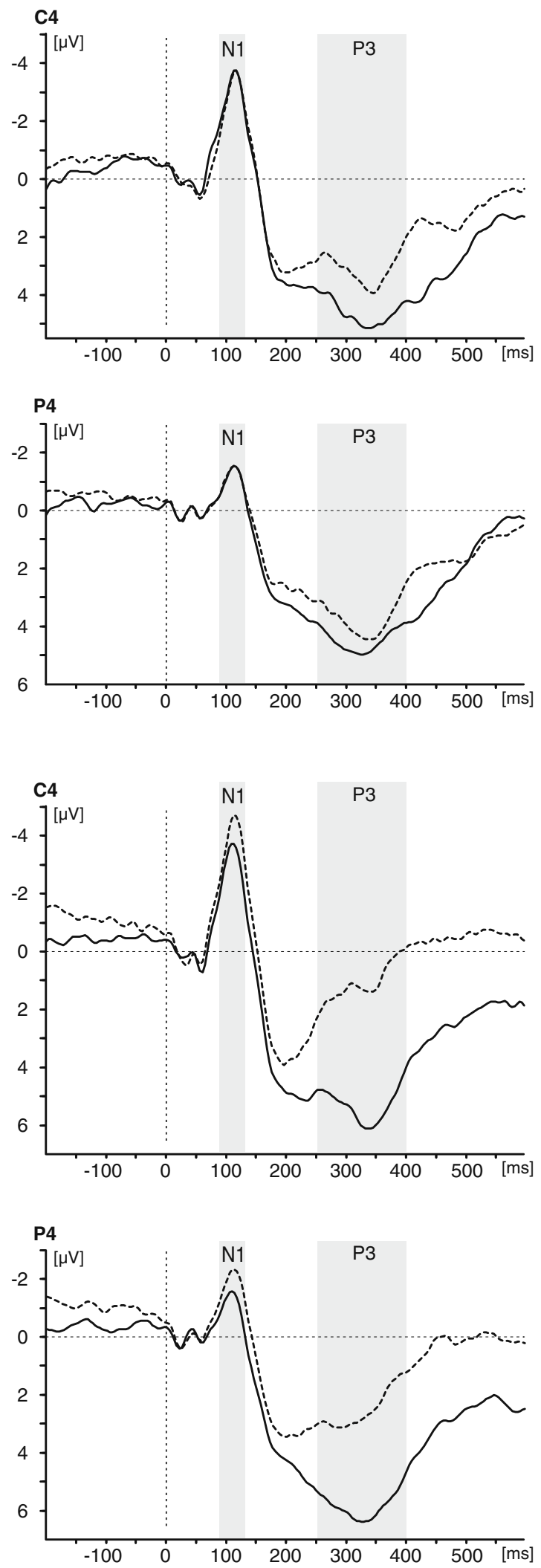
Table 3 Experiment 2: ANOVA on raw and normalized $(M=5$, $S D=2)$ difference amplitudes (ERP[valid] ERP[invalid]) in the time epochs $40 \mathrm{~ms}$ prior to the stimulus and 90-130 mis poststimulus

\begin{tabular}{llllllllll}
\hline Effect & \multicolumn{3}{c}{ Raw Scores } & \multicolumn{5}{c}{ Standardized Scores } \\
\hline & $\mathrm{df}$ & $\mathrm{F}$ & $\mathrm{p}$ & $\varepsilon$ & $\eta^{2}$ & $\mathrm{~F}$ & $\mathrm{p}$ & $\varepsilon$ & $\eta^{2}$ \\
& $2 / 48$ & 6.32 & $<.01$ & 0.73 & .21 & 6.4 & $<.01$ & 0.72 & .21 \\
Interval*PrePost*ACP & $4 / 96$ & 5.75 & $<.01$ & 0.79 & .19 & 5.75 & $<.01$ & 0.79 & .19 \\
Interval*PrePost*ACP*LMR & & & & & & & & & \\
Short Interval & $4 / 96$ & 2.78 & $<.04$ & 0.95 & .10 & 2.63 & $<.05$ & 0.94 & .10 \\
PrePost*ACP*LMR & & & & & & & & & \\
Long Interval & $2 / 48$ & 5.39 & $<.02$ & 0.86 & .18 & 6.77 & $<.01$ & 0.75 & .22 \\
PrePost*ACP & $4 / 96$ & 3.67 & $<.02$ & 0.84 & .13 & 3.9 & $<.01$ & 0.88 & .14 \\
PrePost*ACP*LMR & & & & & & & &
\end{tabular}

\section{Discussion}

Experiment 2 investigated whether temporal orienting enhances early sensory levels of auditory processing when attention is manipulated in a trial-by-trial manner but can be focused selectively on the cued time point. Reaction time data from the behavioral session replicated the findings from Experiment 1 and from earlier visual-cuing studies with a go/no-go task: Participants responded more quickly to valid than to invalid targets, but only for the short interval. Accuracy measures were not affected by validity, suggesting that the reaction time benefit was not due to a speed-accuracy trade-off. Thus, this finding provides behavioral evidence for the validity of auditory temporal orienting in the go/no-go cuing paradigm.

ERP data of Experiment 2 revealed several electrophysiological correlates of selective temporal orienting in the auditory modality. Most important, for the short interval, N1 amplitudes were larger for valid than for invalid nontargets over the left frontal and central scalp, suggesting that selective temporal orienting improves early perceptual

Table 4 Experiment 2: ANOVA on mean amplitudes between 250 and $400 \mathrm{~ms}$. Only significant effects involving the factors validity or interval are displayed

\begin{tabular}{llllll}
\hline Effect & df & F & p & $\varepsilon$ & $\eta^{2}$ \\
\hline Validity & $1 / 24$ & 24.04 & $<.01$ & - & .50 \\
Validity*ACP & $2 / 48$ & 14.52 & $<.01$ & 1.01 & .38 \\
Validity*interval & $1 / 24$ & 26.14 & $<.01$ & - & .52 \\
Validity*interval*LMR & $2 / 48$ & 5.51 & $<.01$ & 0.91 & .19 \\
Validity*interval*ACP*LMR & $4(96$ & 5.61 & $<.01$ & 1.06 & .19 \\
Short Interval & & & & & \\
Validity & $1 / 24$ & 5.21 & .03 & - & .18 \\
Validity*ACP & $2 / 48$ & 7.82 & $<.01$ & 0.94 & .25 \\
Long Interval & & & & & \\
Validity & $1 / 24$ & 30.58 & $<.01$ & - & .56 \\
Validity*ACP & $2 / 48$ & 14.30 & $<.01$ & 1.08 & .37 \\
Validity*LMR & $2 / 48$ & 4.32 & .02 & 0.87 & .15 \\
Validity*ACP*LMR & $4 / 96$ & 4.06 & $<.01$ & 1.05 & .14 \\
\hline
\end{tabular}

stages of auditory processing. The enhanced negativity in the time range of the $\mathrm{N} 1$ is consistent with the findings of studies in which the selective temporal-orienting paradigm was used, where attention was manipulated between blocks (Lange et al., 2006; Lange \& Röder, 2006; Lange et al., 2003; Röder et al., 2007; Sanders \& Astheimer, 2008; for a review, see Lange \& Röder, 2010). The results of our Experiment 2 extend this finding to a paradigm, in which the focus of attention changes between trials. For the long interval, a reversed $\mathrm{N} 1$ effect was observed, as in Experiment 1. This will be addressed in the General Discussion section.

As in Experiment 1, we observed a slow negativity over the frontocentral scalp between the cue and the target/ nontarget. In Experiment 2, however, this negativity was more pronounced prior to the to-be-attended time point and may, thus, be associated with the allocation of attention in time (see also Griffin et al., 2002; Lange et al., 2003; Miniussi et al., 1999; Sanders \& Astheimer, 2008). Thus, CNV-like negativities developed in both experiments, but, just like the enhanced negativity in the N1 time range,effects of temporal orienting were found only in Experiment 2. It is unlikely, though, that the more negative $\mathrm{N} 1$ to attended nontargets was due to the difference in the preceding slow wave. The enhanced N1 was obtained with a strict baseline, set from 0 to $50 \mathrm{~ms}$, which should reduce the impact of the slow wave on the N1 (e.g., Correa et al., 2006; Correa \& Nobre, 2008; Griffin et al., 2002). More important, the attention effect in the slow wave and the N1 attention effect could be separated topographically, thus suggesting that the two effects do not reflect identical processes (see also Lange et al., 2003).

In spatially oriented auditory attention, there are two main interpretations of $\mathrm{N} 1$ attention effects (for a review, see Näätänen \& Alho, 2004). The two approaches differ in whether early processing of attended and unattended stimuli is supposed to differ quantitatively (e.g., Hillyard et al., 1973) or qualitatively (e.g., Näätänen, 1982). Hillyard and colleagues proposed a filter or gatingmechanism (gain control) that modulates the amplitude of the N1 (Hillyard, 1981; Hillyard et al., 1973; Woldorff \& Hillyard, 1991). By 
contrast, Näätänen (e.g., Näätänen, 1982) suggested that the enhanced N1 was actually due to the superposition of an endogenous processing negativity, which was assumed to reflect the additional processing received by stimuli with the attended feature (Näätänen et al., 1978). The spatialorienting processing negativity is a longer lasting effect, the early part of which may overlap with the auditory N1 (e.g., Hansen \& Hillyard, 1980; Näätänen et al., 1978). In earlier auditory studies of temporally selective attention, the enhanced negativity was restricted to the time range of the $\mathrm{N} 1$ itself, which is consistent with the idea of a gating mechanism (e.g., Lange et al., 2006; Lange et al., 2003; Sanders \& Astheimer, 2008). The enhanced negativity observed in the present study is, however, most likely not due to an amplitude modulation of the N1 proper, since it extended somewhat to the time range prior to the N1. The effect is, therefore, rather compatible with an early and short-lived endogenous processing negativity. The shorter duration, as compared with what is known from spatially selective attention (e.g., Hansen \& Hillyard, 1980; Schröger \& Eimer, 1997), may be due to inherent differences in the processing of temporal and spatial information. In temporal orienting, the relevant information concerns the attended time point (i.e., the end of the short or the long interval). This information accumulates during the course of the interval until the target or nontarget occurs. The onset of the target or nontarget indicates the end of the interval, thus completing the processing of temporal information. In spatial orienting, by contrast, the processing of the (spatial) information can start only with the presentation of the stimulus. Thus, although the early attention-related negativity may not necessarily reflect an enhancement of the N1 proper, Experiment 2 nevertheless provides evidence that selective temporal orienting triggered in a trial-by-trial manner is associated with modulations occurring early in the auditoryprocessing stream.

Additionally, an enhancement of the P3-like positivity was found in Experiment 2. By contrast to our Experiment 1 and to other temporal-orienting studies (Griffin et al., 2002; Miniussi et al., 1999; Sanders \& Astheimer, 2008), this attention-related positivity was found in the ERPs to nontargets - that is, to stimuli that did not require a response (see also (Lange et al., 2006; Lange et al., 2003). Therefore, it is unlikely that this effect reflects processes involved in response selection or preparation, as has been suggested for the enhanced positivity to temporally attended targets (e.g., Nobre, 2001). It has recently been suggested that the P3 to no-go stimuli (equivalent to our nontarget stimuli) is an index of motor inhibition (e.g., Bruin, Wijers, \& van Staveren, 2001; Smith, Johnstone, \& Barry, 2006, 2007). In support of this notion, it has been shown that the amplitude of the no-go P3 depends on the level of prior preparation for a response: The stronger a response had been prepared, the more inhibition was necessary to suppress its execution in cases in which a no-go stimulus was presented (Bruin et al., 2001; Smith et al., 2007). The results of Experiment 2 are also in line with this interpretation: Since only stimuli at the cued time point were relevant for response-selection, motor preparation may have been stronger at the cued than at the uncued time point. Hence, to refrain from responding to nontargets may have required more inhibition at the cued than at the uncued time point. This should be reflected in the larger P3 to valid than to invalid nontargets.

\section{General discussion}

The aim of the present study was to gain further insight into how temporal orienting alters auditory processing. Experiment 1 investigated whether the behavioral benefits of auditory temporal orienting may be due to modulations at sensoryprocessing levels. Consistent with an orienting of attention to the cued time point, the participants responded more quickly to cued than to uncued targets of the short interval. This reaction time benefit was not accompanied by modulations of early sensory-processing stages. Rather, ERP effects indicated an involvement of later processing stages, possibly related to stimulus-evaluation or response-selection. Experiment 2 tested whether earlier processing levels are affected by temporal orienting when attention is manipulated in a trial-by-trial manner (as in Experiment 1) but can be focused selectively to the cued time point. In this experiment, only stimuli at the cued time point were relevant for response selection. Here, a modulation in the timerange of the auditory N1 was found, in addition to a later attention-related positivity. However, attention effects cannot be analyzed on a behavioral level when the participants do not respond to the uncued stimuli. Hence, the functional significance of these effects remains to be evaluated.

The behavioral effect obtained with the choice reaction task of Experiment $1\left(\eta^{2}=.26\right)$ was slightly larger than that observed in the go/no-go task of Experiment $2\left(\eta^{2}=.16\right)$. Because the perceptual discrimination demands (i.e., distinguishing between a continuous target and a target with a gap) were identical between the tasks, this pattern is consistent with the notion that the speeded responding to temporally attended targets is partly due to improvements of processes related to response-selection. Also in agreement with this idea are the ERP results for Experiment 1: Effects of temporal orienting were observed only in a late centroparietal positivity, but not in earlier sensory-related deflections. In Experiment 1, all stimuli at the end of the interval were associated with a response (targets). Increased P3 amplitudes for valid, as compared with invalid, targets 
have been reported earlier (Griffin et al., 2002; Miniussi et al., 1999; Sanders \& Astheimer, 2008; but see Correa et al., 2006). This late modulation in the P3-like positivity has been interpreted as evidence that temporal orienting affects later processes, related to stimulus-evaluation or motor preparation (for a review, see, e.g., Nobre, 2001; see also Hillyard et al., 1973, for a similar interpretation of spatial attention effects in the P3). The attention effect in the late positivity was restricted to the short interval in the visual-cuing studies, as was the reaction time effect. By contrast, we observed a similar modulation for targets of the long interval in Experiment 1 of the present study, although a reaction time effect was not observed in this condition. Thus, it remains to be investigated whether and how the enhancement of the late positivity is functionally related to the behavioral benefit for temporally attended targets.

The enhanced N1 observed for nontargets of the short interval in Experiment 2 provides evidence that temporal orienting affects early levels of auditory processing, if attention can be selectively focused on one particular time point. The exact mechanisms underlying this effect remain to be specified (see the Discussion section for Experiment 2 ). The most likely explanation for why an early effect was observed in Experiment 2, but not in Experiment 1, is that, in Experiment 2, only targets at the cued time point were relevant for response selection, whereas both cued and uncued targets required a response in Experiment 1 (see also Eimer, 1994, 1996, for a similar idea in spatial orienting). As a consequence, the participants had to divide their attention between the cued and the uncued moments in Experiment 1. By contrast, they could selectively orient their attention to the cued time point in Experiment 2. Thus, the difference in attention allocated to the cued and the uncued time points may have been larger in Experiment 2 than in Experiment 1. It may be suggested that the larger the difference in attention between two conditions, the earlier the processing levels affected. Additionally, the early effect observed in Experiment 2 might have been favored by the fact that the location of targets and nontargets was constant in Experiment 1 but changed unpredictably between the two ears in Experiment 2 (see Miniussi et al., 1999, for a similar suggestion). Although we cannot rule out completely that perceptual uncertainty contributed to the different result patterns of the two experiments, there is evidence that it is not crucial for early effects to occur: Correa et al. (2006) reported early effects of temporal orienting in the visual modality with a fixed foveal presentation (see also Miniussi, Rao, \& Nobre, 2002).

Notably, Experiment 2 provides evidence that temporal orienting can affect early levels of auditory processing even if attention is manipulated between trials, rather than between blocks. By contrast, in earlier temporal-orienting studies reporting early effects on auditory processing, the attentional focus was constant for a block of trials (Lange et al., 2006; Lange \& Röder, 2006; Lange et al., 2003; Röder et al., 2007; Sanders \& Astheimer, 2008). Since we did not directly manipulate the consistency of the attentional focus in our study, we cannot conclude that it does not influence the locus of attentional selection. Earlier visual studies reported larger behavioral effects of temporal orienting when attention was manipulated between, rather than within, blocks (Correa et al., 2004). Moreover, using a constant attentional focus, Correa et al. (2006) found a modulation of early visual processing even when participants had to divide their attention between cued and uncued time points. Future studies need to investigate directly whether the presence of early effects is further modulated by whether attention is manipulated within or between blocks.

The attention-related enhancement in the timerange of the $\mathrm{N} 1$ for short-interval stimuli is consistent with findings of earlier auditory temporal-orienting studies (for a review, see Lange \& Röder, 2010). By contrast, the reduced N1 to cued versus uncued long-interval stimuli is a new finding, which might be specific to the trial-by-trial cuing task used in the present study. This effect was both unpredicted and contrary to what was expected on the basis of the temporalorienting literature. We can, therefore, only speculate on possible explanations. In earlier experiments, where temporal orienting was induced by a regular (vs. irregular) tone sequence, we also observed an attenuation of the N1-but only when the timing of the target tone was certain (Lange, 2009). When the timing of the target tone was uncertain, an enhancement of the N1 was found (Lange, 2010). Reductions of N1 amplitude may thus be related to temporal predictability or certainty (see also Clementz, Barber, \& Dzau, 2002; Lange, 2009; Rothman, Davis, \& Hay, 1970; Schafer, Amochaev, \& Russell, 1981). In the present study, stimuli at the end of the long interval were temporally highly predictable, because the conditional probability that a stimulus would occur increased with elapsing time. Prior to the ending of the long interval, the participants were almost certain that a stimulus would follow at the end of the long interval. By contrast, prior to the ending of the short interval, the participants did not know whether a stimulus would be presented at the end of the short or at the end of the long interval. Thus, temporal certainty was low for stimuli at the end of a short interval.

The conditional probability is independent of the time interval indicated by the cue. Therefore, increased conditional probability alone does not explain the smaller N1 amplitude for cued, as compared with uncued, long-interval stimuli. Moreover, several earlier studies reported an enhancement of the N1 for long-interval stimuli,even though the participants knew that a stimulus was about to appear (Lange et al., 2006; Lange et al., 2003; Sanders \& Astheimer, 2008). In 
these experiments, the a priori probabilities of attended and unattended stimuli were identical. By contrast, in the present study, the probability for a stimulus at the end of the long interval was higher when the long interval had been cued than when the short interval had been cued. If the cueinduced a priori expectations are not fully reset during the course of the trial (see also Karlin, 1966), this may yield an overall higher predictability of validly cued stimuli at the end of the long interval (although this was not reflected in faster responding).

It may be hypothesized that two kinds of processes are activated in temporal-orienting studies: processes related to temporal orienting and processes related to temporal predictability or certainty. These processes have an opponent influence on the N1: Temporal orienting leads to an enhancement, whereas temporal certainty leads to a reduction. The relative contributions of these processes may vary, depending, for example,on conditional probability and/or overall predictability. An enhancement of the N1 may thus be observed only when the timing of the stimulus is uncertain prior to stimulus delivery. When a participant can be certain that a stimulus will be presented, however, processes leading to an attenuation of the N1 will dominate, which may mask attention-related enhancements.

In the absence of corresponding behavioral data, only limited conclusions concerning the functional significance of N1 modulations can be drawn. The auditory N1 has been associated with processes related to transient detection or to the nonspecific triggering of attention, rather than processes that feed directly to sensory identification (for a review, see Näätänen \& Picton, 1987). In line with this idea, it may be hypothesized that temporal orienting increases the stimuli's attention-triggering properties when the timing of a stimulus is uncertain - possibly to prepare the auditory system for processing the task-relevant stimuli. When stimulus timing is certain before the stimulus occurs, these processes might take place prior to stimulus delivery (see also Lange, in press). As a consequence, any further triggering of attention will be unnecessary at the time the stimulus is actually presented. Testing these assumptions will be a challenge for future studies.

\section{Conclusions}

To summarize the present findings, we were able to show that temporal orienting improves auditory processing, possibly due to modulations at later decision- or responserelated processing stages. Early auditory processing was affected only when attention could be focused selectively on one time point. It remains to be evaluated whether these early modulations also contribute to the behavioral effects.
Acknowledgments The Study was supported by the Deutsche Forschungsgemeinschaft (German Research Foundation) Grant LA2486/1-1. We thank Barbara Reese and Robert Schnürch for their help during data acquisition and Daniela Czernochowski for helpful comments.

\section{References}

Birbaumer, N., Elbert, T., Canavan, A. G. M., \& Rockstroh, B. (1990). Slow potentials of the cerebral cortex and behaviour. Physiological Reviews, 70, 1-41.

Bruin, K. J., Wijers, A. A., \& van Staveren, A. S. J. (2001). Response priming in a go/nogo task: Do we have to explain the go/nogo N2 effect in terms of response activation instead of inhibition? Clinical Neurophysiology, 112, 1660-1671.

Clementz, B. A., Barber, S. K., \& Dzau, J. R. (2002). Knowledge of stimulus repetition affects the magnitude and spatial distribution of low-frequency event-related brain potentials. Audiology and Neuro-Otology, 7, 303-314.

Correa, A., Lupiáñez, J., Madrid, E., \& Tudela, P. (2006). Temporal attention enhances early visual processing: A review and new evidence from event-related potentials. Brain Research, 1076, $116-128$.

Correa, A., Lupiáñez, J., Milliken, B., \& Tudela, P. (2004). Endogenous temporal orienting of attention in detection and discrimination tasks. Perception \& Psychophysics, 66, 264-278.

Correa, A., Lupiáñez, J., \& Tudela, P. (2005). Attentional preparation based on temporal expectancies modulates processing at the perceptual level. Psychonomic Bulletin \& Review, 12, 328-334.

Correa, A., \& Nobre, A. C. (2008). Neural modulation by regularity and passage of time. Journal of Neurophysiology, 100, 1649-1655.

Coull, J. T., \& Nobre, A. C. (1998). Where and when to pay attention: The neural systems for directing attention to spatial locations and to time intervals as revealed by both PET and fMRI. The Journal of Neuroscience, 18, 7426-7435.

Eimer, M. (1994). "Sensory gating" as a mechanism for visuospatial orienting: Electrophysiological evidence from trial-by-trial cuing experiments. Perception \& Psychophysics, 55, 667-675.

Eimer, M. (1996). ERP modulation indicate the selective processing of visual stimuli as a result of transient and sustained spatial attention. Psychophysiology, 33, 13-21.

Elbert, T., Ulrich, R., Rockstroh, B., \& Lutzenberger, W. (1991). The processing of temporal Intervals reflected by CNV-like brain potentials. Psychophysiology, 28, 648-655.

Giard, M. H., Perrin, F., Pernier, J., \& Peronnet, F. (1988). Several attention-related wave forms in auditory areas: A topographic study. Electroencephalography and Clinical Neurophysiology, 69, 371-384.

Gratton, G., Coles, M. G. H., \& Donchin, E. (1983). A new method for off-line removal of ocular artifact. Electroencephalography and Clinical Neurophysiology, 55, 468-484.

Griffin, I. C., Miniussi, C., \& Nobre, A. C. (2001). Orienting attention in time. Frontiers in Bioscience, 6, 660-671.

Griffin, I. C., Miniussi, C., \& Nobre, A. C. (2002). Multiple mechanisms of selective attention: Differential modulation of stimulus processing by attention to space or time. Neuropsychologia, 40, 2325-2340.

Hansen, J. C., \& Hillyard, S. A. (1980). Endogenous brain potentials associated with selective auditory attention. Electroencephalography and Clinical Neurophysiology, 49, 277-290.

Hillyard, S. A. (1981). Selective auditory attention and early eventrelated potentials-Arejoinder. Canadian Journal of Psychology, $35,159-174$. 
Hillyard, S. A., Hink, R., Schwent, V. L., \& Picton, T. (1973). Electrical signs of selective attention in the human brain. Science, 162, 177-180.

Huynh, H., \& Feldt, L. S. (1976). Estimation of the box correction for degrees of freedom from sample data in randomized block and splitsplot designs. Journal of Educational Statistics, 1, 69-82.

Karlin, L. (1966). Development of readiness to respond during short foreperiods. Journal of Experimental Psychology, 72, 505-509.

Lange, K. (2009). Brain correlates of early auditory processing are attenuated by expectations for time and pitch. Brain and Cognition, 69, 127-137.

Lange, K. (2010). Can a regular context induce temporal orienting to a target sound? International Journal of Psychophysiology, 78, 231-238.

Lange, K. (in press). The reduced N1 to self-generated tones: An effect of temporal predictability? Psychophysiology.

Lange, K., Krämer, U. M., \& Röder, B. (2006). Attending points in time and space. Experimental Brain Research, 173, 130-140.

Lange, K., \& Röder, B. (2006). Orienting attention to points in time improves stimulus processing both within and across modalities. Journal of Cognitive Neuroscience, 18, 715-729.

Lange, K., \& Röder, B. (2010). Temporal orienting in audition, touch, and across modalities. In A. C. Nobre \& J. T. Coull (Eds.), Attention and time (pp. 393-405). Oxford: Oxford University Press.

Lange, K., Rösler, F., \& Röder, B. (2003). Early processing stages are modulated when auditory stimuli are presented at an attended moment in time: An event-related potential study. Psychophysiology, 40, 806817.

Macar, F., \& Besson, M. (1985). Contingent negative variation in processes of expectancy, motor preparation and time estimation. Biological Psychology, 21, 293-307.

Mangun, G. R., \& Hillyard, S. A. (1991). Modulations of sensoryevoked brain potentials indicate changes in perceptual processing during visual-spatial priming. Journal of Experimental Psychology. Human Perception and Performance, 17, 1057-1074.

McAdam, D. W. (1967). Development of the contingent negative variation during the learning of a temporal interval. Electroencephalography and Clinical Neurophysiology, 23, 491-494.

McCarthy, G., \& Wood, C. C. (1985). Scalp distributions of event-related potentials: An ambiguity associated with analysis of variance models. Electroencephalography and Clinical Neurophysiology, 62, 203-208.

Miniussi, C., Rao, A., \& Nobre, A. C. (2002). Watching where you look: Modulation of visual processing of foveal stimuli by spatial attention. Neuropsychologia, 40, 2448-2460.

Miniussi, C., Wilding, E. L., Coull, J. T., \& Nobre, A. C. (1999). Orienting attention in time: Modulation of brain potentials. Brain, 122, 1507-1518.

Näätänen, R. (1982). Processing negativity: An evoked-potential reflection of selective attention. Psychological Bulletin, 92, 605-640.

Näätänen, R., \& Alho, K. (2004). Mechanisms of attention in audition as revealed by the event-related potentials of the brain. In M. I. Posner (Ed.), Cognitive neuroscience of attention (pp. 194-206). New York: Guilford.

Näätänen, R., Gaillard, A. W. K., \& Mäntysalo, S. (1978). Early selective-attention effect on evoked potential reinterpreted. Acta Psychologica, 42, 313-329.

Näätänen, R., \& Picton, T. (1987). The N1 wave of the human electric and magnetic response to sound: A review and an analysis of the component structure. Psychophysiology, 24, 375-425.

Nobre, A. C. (2001). Orienting attention to instants in time. Neuropsychologia, 39, 1317-1328.

Nobre, A. C. (2004). Probing the flexibility of attentional orienting in the human brain. In M. I. Posner (Ed.), Cognitive neuroscience of attention (pp. 157-179). New York: Guilford.
Nobre, A. C., Correa, A., \& Coull, J. T. (2007). The hazards of time. Current Opinion in Neurobiology, 17, 465-470.

Nobre, A. C., \& Coull, J. T. (2010). Attention and time. Oxford: Oxford University Press.

Nobre, A. C., \& O'Reilly, J. (2004). Time is of the essence. Trends in Cognitive Sciences, 8, 387-389.

O'Brien, R. G., \& Kaiser, M. K. (1985). MANOVA for analyzing repeated measurement design: An extensive primer. Psychological Bulletin, 97, 316-333.

Posner, M. I., Snyder, C. R. R., \& Davidson, B. J. (1980). Attention and the detection of signals. Journal of Experimental Psychology. General, 109, 160-174.

Pouthas, V. (2003). Electrophysiological evidence for specific processing of temporal information in humans. In W. H. Meck (Ed.), Functional and neural mechanisms of interval timing (pp. 439-456). Boca Raton: CRC Press.

Pouthas, V., Garnero, L., Ferrandez, A. M., \& Renault, B. (2000). ERPs and PET analysis of time perception: Spatial and temporal brain mapping during visual discrimination tasks. Human Brain Mapping, 10, 49-60.

Röder, B., Krämer, U. M., \& Lange, K. (2007). Congenitally blind humans use different stimulus selection strategies in hearing: An ERP study of spatial and temporal attention. Restorative Neurology and Neuroscience, 25, 311-322.

Rothman, H. H., Davis, H., \& Hay, I. S. (1970). Slow evoked cortical potentials and temporal features of stimulation. Electroencephalography and Clinical Neurophysiology, 29, 225-232.

Ruchkin, D. S., McCalley, M. G., \& Glaser, E. M. (1977). Event related potentials and time estimation. Psychophysiology, 14, 451-455.

Sanders, L. D., \& Astheimer, L. B. (2008). Temporally selective attention modulates early perceptual processing: Event-related potential evidence. Perception \& Psychophysics, 70, 732-742.

Schafer, E. W. P., Amochaev, A., \& Russell, M. J. (1981). Knowledge of stimulus timing attenuates human evoked cortical potentials. Electroencephalography and Clinical Neurophysiology, 52, 9-17.

Schröger, E., \& Eimer, M. (1997). Endogenous covert spatial orienting in audition: "Cost-benefit" analysis of reaction times and event-related potentials. The Quarterly Journal of Experimental Psychology, 50A, 457-474.

Smith, J. L., Johnstone, S. J., \& Barry, R. J. (2006). Effects of prestimulus processing, on subsequent events in a warned go/nogo paradigm: Response preparation, execution and inhibition. International Journal of Psychophysiology, 61, 121-133.

Smith, J. L., Johnstone, S. J., \& Barry, R. J. (2007). Response priming in the go/nogo task: The N2 reflects neither inhibition nor conflict. Clinical Neurophysiology, 118, 343-355.

Talsma, D., Mulckhuyse, M., Slagter, H. A., \& Theeuwes, J. (2007). Faster, more intense! The relation between electrophysiological reflections of attentional orienting, sensory gain control, and speed of responding. Brain Research, 1178, 92-105.

Talsma, D., Slagter, H. A., Nieuwenhuis, S., Hage, J., \& Kok, A. (2005). The orienting of visuospatial attention: An event-related brain potential study. Cognitive Brain Research, 25, 117-129.

Urbach, T. P., \& Kutas, M. (2002). The intractability of scaling scalp distributions to infer neuroelectric sources. Psychophysiology, 39, 791-808.

Walter, W. G., Winter, A. L., Cooper, R., McCallum, W. C., \& Aldridge, V. J. (1964). Contingent negative variation electric sign of sensorimotor association+expectancy in human brain. Nature, 203, 380-384.

Woldorff, M. G., \& Hillyard, S. A. (1991). Modulation of early auditory processing during selective listening to rapidly presented tones. Electroencephalography and Clinical Neurophysiology, 79, 170-191. 